\title{
Inelastic scattering of fast neutrons from ${ }^{56} \mathrm{Fe}$
}

\author{
Roland Beyer, a, Evert Birgersson ${ }^{1, b}$, Mirco Dietz ${ }^{1,2}$, Roland Hannaske ${ }^{1,2, c}$, Arnd R. Junghans ${ }^{1}$, Toni Kögler ${ }^{1,2}$, \\ Ralph Massarczyk ${ }^{1,2, \mathrm{~d}}$, Andrija Matić ${ }^{1, \mathrm{e}}$, Ronald Schwengner ${ }^{1}$, and Andreas Wagner ${ }^{1}$ \\ 1 Helmholtz-Zentrum Dresden - Rossendorf, 01328 Dresden, Germany \\ 2 Technische Universität Dresden, 01062 Dresden, Germany
}

\begin{abstract}
The inelastic scattering of fast neutrons on ${ }^{56} \mathrm{Fe}$ was investigated in different manners at the neutron time-of-flight facility $n$ ELBE. The scattering cross section was determined via the measurement of the $\gamma$-ray production and by means of a kinematically complete double time-of-flight method. In a further measurement the $\gamma$-ray angular distribution was determined to correct the measured cross sections for anisotropy. The resulting inelastic scattering cross section determined from the photo production cross sections is in very good agreement with evaluations and previous measurements. In contrast, the result of the double time-offlight measurement is about $10 \%$ lower than these data, giving a hint to neutron- $\gamma$-ray angular correlations in the process of inelastic neutron scattering.
\end{abstract}

\section{Introduction}

The CIELO project (Collaborative International Evaluated Library Organization) [1] selected six highest-priority pilot isotopes to initially be investigated. Besides ${ }^{1} \mathrm{H},{ }^{16} \mathrm{O},{ }^{235} \mathrm{U}$, ${ }^{238} \mathrm{U}$ and ${ }^{239} \mathrm{Pu}$ there is ${ }^{56} \mathrm{Fe}$ which is one of the major construction material of nuclear facilities. This importance was already mentioned in the context of the development of Generation IV nuclear reactors and transmutation technologies $[2,3]$ as well as by the OECD NEA high priority request list [4]. All these references identified inelastic neutron scattering as one important nuclear interaction that should be known to higher accuracy than offered by current evaluation libraries. In the neutron energy range from several up to a few $\mathrm{MeV}$ the target uncertainties are lower than 5\%.

The inelastic scattering of fast neutrons has been measured for several decades applying two different methods: 1. Using quasi-monoenergetic neutron beams and detecting the scattered neutron or 2. employing a continuous neutron spectrum and detecting the de-excitation $\gamma$-rays. For iron the former method was used e.g. in Refs. [5-8]. The advantage of this technique is the very good discrimination between different excited states of the target isotope and the simultaneous measurement of the elastic scattering cross section. The drawback is the almost unfeasible high time consumption to scan a wide neutron energy range with small step width. The second method, also called $\gamma$-ray production measurement, e.g. [9-11], is able to deliver cross section data over a wide energy range in a single beam time but causes ambiguities

\footnotetext{
a e-mail: roland. beyer@hzdr.de

b Present address: AREVA NP GmbH, 91052 Erlangen, Germany

${ }^{c}$ Present address: MLUL - Land Brandenburg, 14467 Potsdam, Germany

d Present address: Los Alamos National Laboratory, Los Alamos, New Mexico 87545, USA

e Present address: IBA Proton Therapy, 45157 Essen, Germany
}

if the energy is high enough to excite several nuclear levels. Corrections for branching and feeding increase the resulting uncertainties.

A kinematically complete measurement, i.e. a coincident measurement of the scattered neutron and the deexcitation $\gamma$-ray, can be performed with a continuous energy spectrum and is independent of the knowledge about the level and de-excitation schema of the target nucleus. Therefore, such type of experiment could add further information to the evaluation process and reduce the overall uncertainty of the combined data from all different methods.

\section{The $n$ ELBE neutron time-of-flight facility}

The neutron time-of-flight (ToF) facility $n$ ELBE is the first photo-neutron source at a superconducting electron accelerator, which allows a very precise time structure of the neutron pulses and also favorable background conditions due to the low instantaneous neutron flux and the absence of any moderating materials. At $n \mathrm{ELBE}$ an electron beam of about $30 \mathrm{MeV}$ kinetic energy is focused onto a liquid lead target to produce bremsstrahlung that subsequently produces neutrons via photo-nuclear reactions on the lead nuclei. The short ( $5 \mathrm{ps})$ micropulse length and variable repetition rate (typically $25-400 \mathrm{kHz}$ ) of the electron beam of the ELBE accelerator $[12,13]$ together with a very compact neutron producing target (thickness $11 \mathrm{~mm}$ ) [14] allows us to use a short flight path $(5$ to $11 \mathrm{~m})$ and a correspondingly high neutron intensity. With such a neutron producing target the energy resolution in the fast neutron range is dominated by the achievable ToF resolution of the detectors used. The response functions of different neutron $\mathrm{ToF}$ facilities are discussed in detail in the review article by Schillebeeckx et al. [15]. 


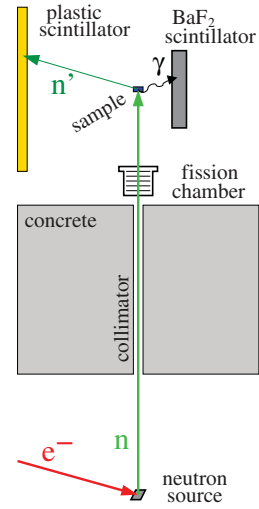

(a)

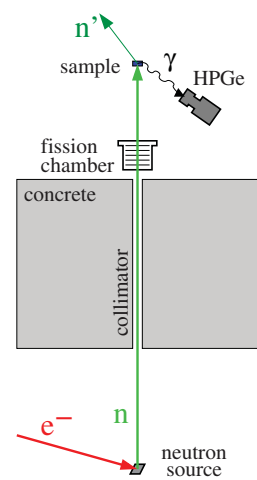

(b)

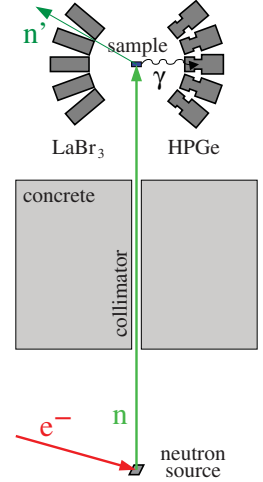

(c)
Figure 1. Schematic view of the experimental setup for the double time-of-flight (a), the $\gamma$-ray production (b) and the $\gamma$-ray angular distribution measurement (c). See text for details.

The $n$ ELBE neutron spectrum ranges from about $10 \mathrm{keV}$ up to $10 \mathrm{MeV}$. The source strength is typically around $2 \cdot 10^{11} \mathrm{n} / \mathrm{s}$ which scales down to a neutron flux of approximately $3 \cdot 10^{4} \mathrm{n} / \mathrm{cm}^{2} / \mathrm{s}$ at the sample position. Further properties of the $n \mathrm{ELBE}$ neutron beam are described in detail in Ref. [16].

\section{The double-ToF experiment}

At $n$ ELBE a detector setup was built to perform kinematically complete measurements of inelastic scattering reactions. A schematic view is shown in Fig. 1(a). The setup consists of five plastic scintillation detectors to detect the scattered neutrons and sixteen $\mathrm{BaF}_{2}$ scintillation detectors to register the de-excitation $\gamma$-rays. The plastic scintillators are made from the material EJ-200, are $1 \mathrm{~m}$ long and have a rectangular cross section of $11 \times 42 \mathrm{~mm}^{2}$ [17]. The $\mathrm{BaF}_{2}$ scintillators are $40 \mathrm{~cm}$ long and have a hexagonal shape with an inner diameter of $53 \mathrm{~cm}$. The sample was a cylinder of pure iron of natural isotopical composition with a diameter of $20 \mathrm{~mm}$ and a length of $8 \mathrm{~mm}$ and was placed at a flight path of $618 \mathrm{~cm}$ from the neutron source. The $\mathrm{BaF}_{2}$ scintillators were arranged in two planes centered above and below the sample position having a mean distance of about 23 to $39 \mathrm{~cm}$ from the sample position. The plastic scintillators form a wall whose center had a distance of $1 \mathrm{~m}$ from the sample. The ${ }^{235} \mathrm{U}$ fission chamber $\mathrm{H} 19$ $[18,19]$ lent by the Physikalisch Technische Bundesanstalt (PTB), Braunschweig, Germany, was used to determine the incoming neutron flux.

The inelastic scattering cross section is calculated from the number of events $N_{\text {det }}$ when one plastic scintillation detector registered a neutron in coincidence with a $\gamma$-ray detected in one of the $\mathrm{BaF}_{2}$ scintillators. These have to be within a so-called kinematic window, which is valid for a certain nuclear level of the target nucleus. These windows are illustrated in Fig. 2 where all coincidence events are plotted. As abscissa the ToF of the incoming neutron $T o F_{\text {in }}$ from the neutron source to the sample is plotted and as ordinate the ToF of the scattered neutron $T o F_{\text {out }}$ from the sample to the plastic scintillator. $T o F_{\text {in }}$ is determined from the time difference between the $\gamma$-ray detection in the $\mathrm{BaF}_{2}$ scintillator and the accelerator reference timing signal. The $T o F_{\text {out }}$ is the time difference

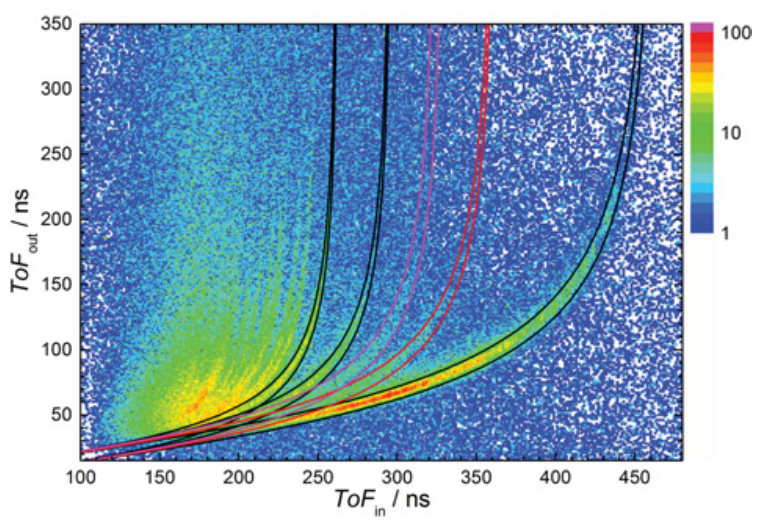

Figure 2. Double time-of-flight histogram of a natural iron sample. The time-of-flight of the incoming neutron $T o F_{\text {in }}$ is determined by the time of the $\gamma$-ray detection and the timeof-flight of the scattered neutron $T o F_{\text {out }}$ is given by the time difference between the neutron and the $\gamma$-ray detection. The solid lines mark the kinematic windows of the inelastic scattering under excitation of the first three levels of ${ }^{56} \mathrm{Fe}$ (black), the first level of ${ }^{54} \mathrm{Fe}$ (red) and the double scattering on two different ${ }^{56} \mathrm{Fe}$ nuclei (magenta).

between the neutron detection in the plastic and the $\gamma$-ray detection in the $\mathrm{BaF}_{2}$ scintillator. The relative time scale is given by the dispersion, i.e. the channel-to-time relation, of the time-to-digital converter (TDC). The absolute timing is done using the position of the $\gamma$-flash and the known flight paths.

Figure 2 shows all coincidence events registered during the irradiation of the iron sample. Several bent structures are visible that can be assigned to the inelastic scattering under excitation of different excited states in ${ }^{56} \mathrm{Fe}$. A signature of the first excited state of ${ }^{54} \mathrm{Fe}$ (which accounts to $5.8 \%$ to the natural composition of the sample) is also visible as well as events where a single neutron undergoes two scatterings on two different ${ }^{56} \mathrm{Fe}$ nuclei with excitation of the particular first level. The structures can be analyzed by means of the kinematic windows. These windows are calculated by basic kinematic calculations starting with energy and momentum conservation and taking into account the known masses and level structure of the target nuclei, the neutron mass and the flight paths. The width of the windows are defined by the time resolution of the different detectors.

Summing all events $N_{\text {det }}$ within a kinematic window at a certain $T o F_{\text {in }}$ enables the determination of the inelastic level cross section at a certain neutron energy via the formula:

$$
\sigma_{i j}=\frac{N_{\mathrm{det}}}{\varepsilon_{\mathrm{n}} \cdot \varepsilon_{\gamma} \cdot f_{\text {trans }, \gamma} \cdot W_{i j}} \cdot \frac{1}{\Phi_{\mathrm{n}} \cdot f_{\text {trans }, \mathrm{n}} \cdot A_{\mathrm{targ}}} \cdot \frac{1}{N_{\mathrm{Fe}-56}}
$$

Here $i$ and $j$ denote the detector numbers of the plastic and the $\mathrm{BaF}_{2}$ scintillation detector, respectively, i.e. from every combination of one plastic and one $\mathrm{BaF}_{2}$ scintillator an individual value of the cross section can be determined. The final value is calculated by the arithmetic mean of all detector combinations: $\sigma=\sum_{i, j} \sigma_{i j} /\left(n_{i} \cdot n_{j}\right) . N_{\mathrm{det}}$ already includes a sample-out background subtraction and a ToF-dependent dead time correction. The incoming neutron fluence density $\Phi_{\mathrm{n}}$ in units of $\mathrm{n} / \mathrm{cm}^{2}$ was determined by the ${ }^{235} \mathrm{U}$ fission chamber and normalized 


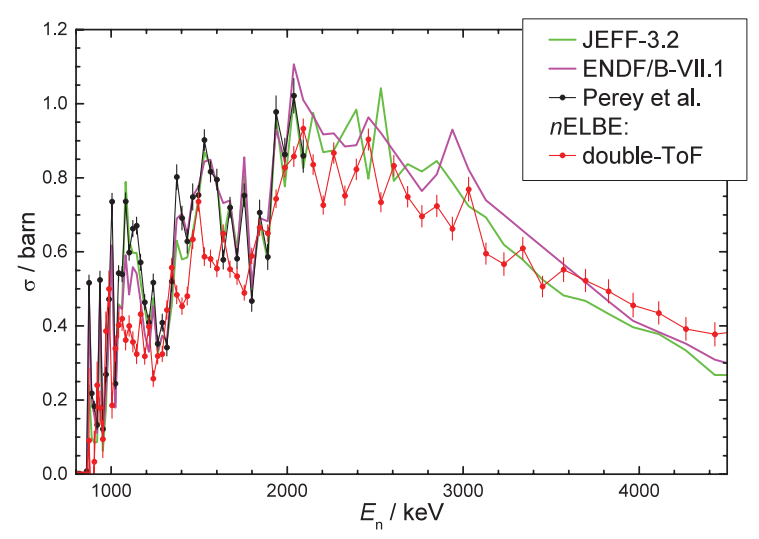

Figure 3. Inelastic neutron scattering cross section under excitation of the first excited state of ${ }^{56} \mathrm{Fe}$. The result of the double-ToF measurement performed at $n \mathrm{ELBE}$ is compared to evaluated data and to the measurement by Perey et al. [9] (Note: The reference data are averaged to the binsize of the $n \mathrm{ELBE}$ measurement.)

to the beam area at the sample position as described in [16]. The neutron detection efficiency $\varepsilon_{\mathrm{n}}$ was determined using quasi-monoenergetic neutrons at the PTB [17]. The total $\gamma$-ray detection efficiency $\varepsilon_{\gamma}$ of the $\mathrm{BaF}_{2}$ detectors was determined in situ using a ${ }^{54} \mathrm{Mn}$ reference source. The transmission of the incoming neutrons from the fission chamber to the sample and the transmission of the emitted $\gamma$-rays from their point of creation to the $\mathrm{BaF}_{2}$ scintillator was determined by Monte-Carlo simulations using MCNP5 [20] and Geant4 [21], respectively. The correction factors $W_{i j}$ for the angular distribution was for now set to 1 assuming isotropic and uncorrelated distributions. $A_{\text {targ }}$ and $N_{\mathrm{Fe}-56}$ denote the sample area and the number of target nuclei in the sample.

Figure 3 shows the result of these calculations. The cross section determined via the double-ToF method is compared to the values taken from the JEFF-3.2 [22] and ENDF/B-VII.1 [23] evaluated data and the previous measurement by Perey et al. [9]. Note that for better visibility the reference data were averaged to this work's binsize. One can see that the double-ToF results follow the same trend as the other data but the absolute values are in average $20 \%$ lower. The contribution of angular distributions to these discrepancies will be discussed in Sect. 6.

\section{The $\gamma$-ray production measurement}

Parallel to the double-ToF measurement a $\gamma$-ray production measurement was performed. Details and results of this measurement were already published in Ref. [24].

A single high-purity germanium (HPGe) detector was mounted between the $\mathrm{BaF}_{2}$ detectors used for the doubleToF measurement described in the previous section. A schematic view is shown in Fig. 1(b). The HPGe detector had a relative efficiency of $100 \%$ and was positioned at a distance of $20 \mathrm{~cm}$ from the sample and at an angle of $125^{\circ}$ relative to the incoming neutron beam.

The $\gamma$-ray production measurement of the inelastic neutron scattering is based on the detection of only the deexcitation $\gamma$-rays. The energy of the incoming neutron is determined by the time of the $\gamma$-ray detection relative to the accelerator reference signal. The excited state of the

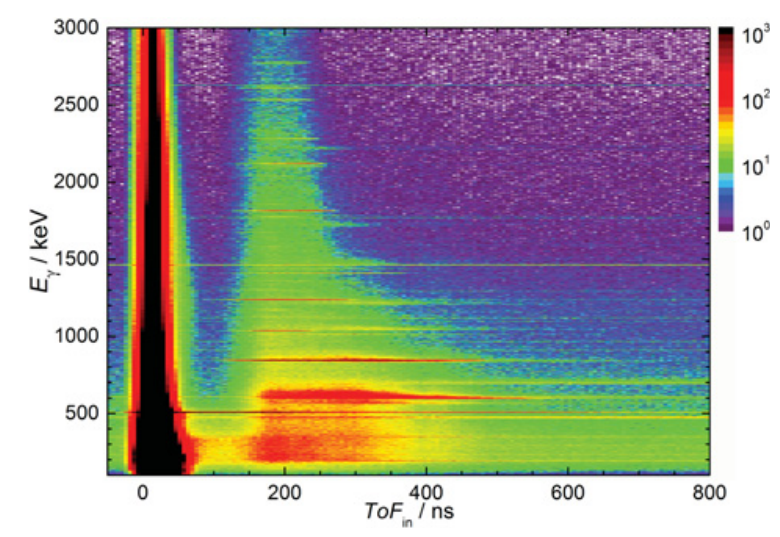

Figure 4. Distribution of events registered by the HPGe detector in dependency of their time-of-flight and their $\gamma$-ray energy.

target nucleus is identified by the energy of the $\gamma$-ray. Only a HPGe detector is able to deliver this information due to its superior energy resolution. The disadvantage of this detector type is its poorer time resolution. Therefore and due to the short flight path between the neutron producing target and the sample the achievable energy resolution is much worse compared to the double-ToF method.

Figure 4 shows the energy-time correlation plot accumulated with the HPGe detector. The very prominent structure at a ToF of about $20 \mathrm{~ns}$ is the $\gamma$-flash caused by the bremsstrahlung emitted by the neutron producing target. Its width of about $10 \mathrm{~ns}$ (FWHM) corresponds to the time resolution of the detector while the contribution from the electron bunch width of less than $10 \mathrm{ps}$ is negligible. The region to the right of the $\gamma$-flash corresponds to the ToF range of the neutrons. Sharp horizontal lines correspond to single nuclear transitions of the target nucleus as well as background radiation, e.g. the $1460 \mathrm{keV}{ }^{40} \mathrm{~K}$ line or the $511 \mathrm{keV}$ line from electron-positron-annihilation. Signatures from inelastic neutron scattering inside the detector are also visible as smeared structures especially below $1 \mathrm{MeV} \gamma$-ray energy.

The different lines that can be assigned to transition between nuclear levels of ${ }^{56} \mathrm{Fe}$ are analyzed as follows. The $2 \mathrm{D}$ histogram is projected onto the $\gamma$-ray energy axis applying narrow $\mathrm{ToF}$ windows, i.e. energy windows corresponding to different incoming neutron energies. From the resulting energy spectra the number of detected $\gamma$-rays $N_{\text {det }}\left(E_{\gamma}, E_{\mathrm{n}}\right)$ can be extracted by fitting Gaussian peak functions. The angle-integrated $\gamma$-ray production cross section can be determined by:

$$
\sigma_{E_{\gamma}}=\frac{N_{\text {det }} \cdot\left(1-p_{\text {mult }}\right)}{\varepsilon_{\gamma} \cdot f_{\text {trans }, \gamma} \cdot W(\theta)} \cdot \frac{1}{\Phi_{\mathrm{n}} \cdot f_{\text {trans }, \mathrm{n}} \cdot A_{\text {targ }}} \cdot \frac{1}{N_{\mathrm{Fe}-56}}
$$

The $\gamma$-ray photon-peak detection efficiency $\varepsilon_{\gamma}$ was measured using standard calibration sources $\left({ }^{22} \mathrm{Na},{ }^{60} \mathrm{Co}\right.$ and $\left.{ }^{226} \mathrm{Ra}\right)$. The incoming neutron fluence $\Phi_{\mathrm{n}}$ was determined as described in the previous section using the PTB fission chamber H19. The correction factors $p_{\text {mult }}$, $f_{\text {trans, } \gamma}$ and $f_{\text {trans,n }}$ for multiple scattering, transmission of the emitted $\gamma$-rays through the sample and transmission of the neutrons from the fission chamber to the target, respectively, were simulated using the Monte-Carlo simulation framework Geant4 [21]. $A_{\text {targ }}$ and $N_{\mathrm{Fe}-56}$ denote the sample area and the number of target nuclei in the sample. The correction factors $W(\theta)$ for the 


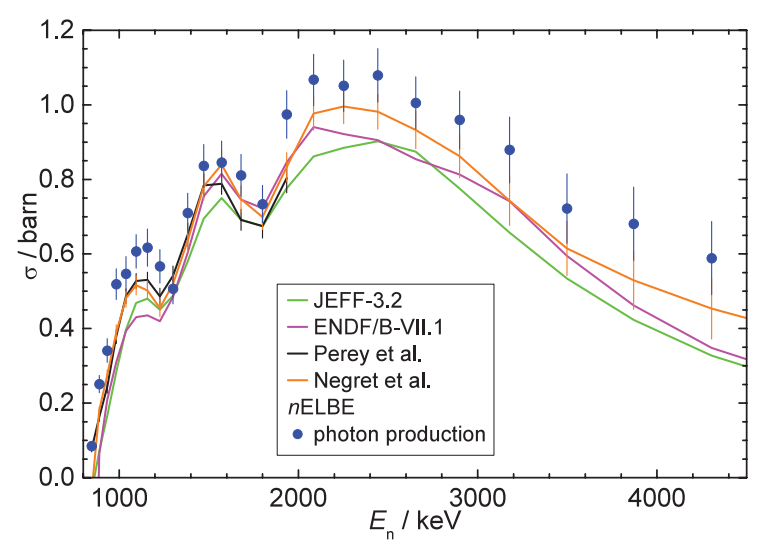

Figure 5. Inelastic neutron scattering cross section under excitation of the first excited state of ${ }^{56} \mathrm{Fe}$. The result of the $\gamma$-ray production measurement performed at $n \mathrm{ELBE}$ is compared to evaluated data and to the measurement by Perey et al. [9] and Negret et al. [11]. (Note: The reference data are averaged to the binsize of the $n$ ELBE measurement.)

angular distribution was set to 1 assuming isotropic $\gamma$-ray emission.

For neutron energies up to the second excited state of the target nucleus the $\gamma$-ray production cross section $\sigma_{E_{\gamma}}$ is identical to the level cross section $\sigma_{E_{x}}$, i.e. the cross section for inelastic scattering under excitation of a certain nuclear level x. Above the threshold of higher lying state it is possible that the de-excitation happens via a cascade producing the same $\gamma$-ray energy like the de-excitation of the lower lying states. E.g. the second excited state of ${ }^{56} \mathrm{Fe}$ decays to $100 \%$ to the first excited state. This feeding and branching has to be taken into account in the determination of level cross section: $\gamma$-ray production cross sections of all transitions feeding a certain state have to be subtracted from the sum of all production cross sections depopulating that state:

$$
\sigma_{E_{x}}=\sum_{\text {depop }} \sigma_{E_{\gamma}, i}-\sum_{\text {feed }} \sigma_{E_{\gamma}, j}
$$

In Fig. 5 the result of this procedure is shown for the first excited state of ${ }^{56} \mathrm{Fe}$. The comparison to the evaluated data and previous measurements reveals a good agreement of the course but a slight up-shift by about $5-15 \%$. The reason for this deviation is caused by the assumption of isotropy as will be resolved in Sect. 6.

\section{The angular distribution measurement}

In a second beam time the angular distribution of the de-excitation $\gamma$-rays of the inelastic scattering on ${ }^{56} \mathrm{Fe}$ was determined. Detailed information about this measurement can be found in another contribution to this proceedings [25].

A setup of $5 \mathrm{LaBr}_{3}$ and 5 HPGe detectors was built. A schematic view is shown in Fig. 1(c). The sample was a cylindrical disk of natural iron with $4.5 \mathrm{~mm}$ thickness and $79 \mathrm{~mm}$ diameter and it was positioned at a flight path of $830 \mathrm{~cm}$. The detectors were arranged in a horizontal plane with a distance of their front faces of $30 \mathrm{~cm}$ to the target. One detector of each type, $\mathrm{LaBr}_{3}$ and HPGe, was placed at angles of $30^{\circ}, 55^{\circ}, 90^{\circ}, 125^{\circ}$ and $150^{\circ}$ with respect to the incoming neutron beam.

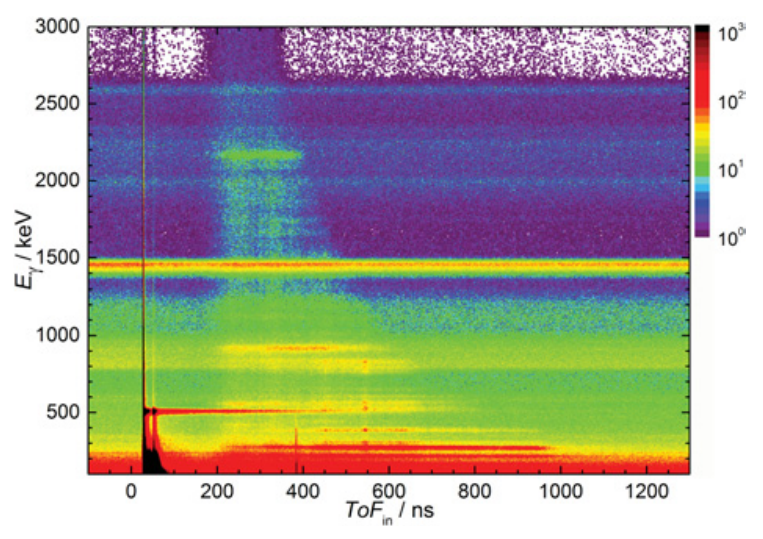

Figure 6. $\gamma$-ray energy versus neutron time-of-flight measured with a $\mathrm{LaBr}_{3}$ detector at an angle of $55^{\circ}$.

During the measurement list-mode data containing the pulse height or signal charge, depending on the detector type, in correlation with the timing information for each detector was acquired. In Fig. 6 an example for the resulting $E_{\gamma}-T o F_{\text {in }}$-correlation plots are shown for a $\mathrm{LaBr}_{3}$ scintillation detector. The histogram for a HPGe detector looks similar to that shown in Fig. 4. The advantages and disadvantage of each detector are clearly visible. The width of the $\gamma$-flash at $T o F_{\text {in }}$ of about 20 to $30 \mathrm{~ns}$ reveals the good time resolution of $\mathrm{LaBr}_{3}$ of about $0.7 \mathrm{~ns}$ (FWHM) compared to the rather poor value for HPGe detector of approximately $10 \mathrm{~ns}$. On the other hand, the $\gamma$-ray energy resolution of the HPGe detector is superior to that one of the $\mathrm{LaBr}_{3}$, e.g. at the energy of the de-excitation $\gamma$-ray of the first excited state of ${ }^{56} \mathrm{Fe}$ of $847 \mathrm{keV}$ the HPGe detector shows $0.15 \%$ resolution and the $\mathrm{LaBr}_{3}$ only $1.3 \%$.

The two-dimensional histograms were analyzed to determine the production yield at a certain angle relative to the one at $90^{\circ}$. Therefore efficiency and energy calibration were done using standard calibration sources $\left({ }^{60} \mathrm{Co},{ }^{88} \mathrm{Y}\right.$ and ${ }^{137} \mathrm{Cs}$ ), background subtraction was done by means of sample-in/sample-out measurements and $\gamma$-ray absorption and extended sample effects were corrected using Geant 4 simulations. From Eq. (2) one can see that neutron beam and sample properties completely cancel out when calculating the ratio of angle-integrated cross sections:

$$
\frac{\sigma(\theta)}{\sigma\left(90^{\circ}\right)}=\frac{N_{\operatorname{det}}(\theta) \cdot f_{\text {corr }}(\theta)}{W(\theta)} \cdot \frac{W\left(90^{\circ}\right)}{N_{\operatorname{det}}\left(90^{\circ}\right) \cdot f_{\text {corr }}\left(90^{\circ}\right)}
$$

The correction factors $f_{\text {corr }}(\theta)$ combine all corrections, which are different for the individual detectors, i.e. efficiency, $\gamma$-ray absorption, multiple scattering etc. The angle-integrated $\gamma$-ray production cross section should be independent of the detector position, i.e. $\frac{\sigma(\theta)}{\sigma\left(90^{\circ}\right)} \equiv 1$. Therefore one can define and determine the normalized angular distribution $W_{\mathrm{n}}(\theta)$ by:

$$
W_{\mathrm{n}}(\theta):=\frac{W(\theta)}{W\left(90^{\circ}\right)}=\frac{N_{\mathrm{det}}(\theta) \cdot f_{\mathrm{corr}}(\theta)}{N_{\operatorname{det}}\left(90^{\circ}\right) \cdot f_{\operatorname{corr}}\left(90^{\circ}\right)}
$$

The angular distribution $W(\theta)$ can be expressed in Legendre polynomials,

$$
W(\theta)=1+a_{2} P_{2}(\cos \theta)+a_{4} P_{4}(\cos \theta)+\cdots
$$

In most cases it is sufficient to use a maximum polynomial order of only 4 to describe the experimental data. From 


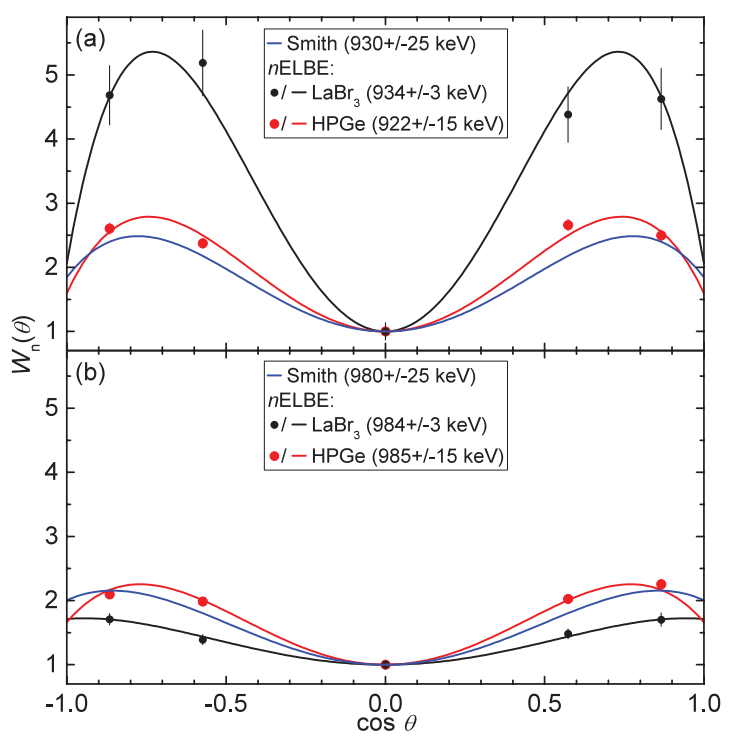

Figure 7. Measured (dots) and fitted (lines) $\gamma$-ray angular distribution of the $E_{\gamma}=847 \mathrm{keV}$ transition of ${ }^{56} \mathrm{Fe}$ at $E_{n} \approx$ $930 \mathrm{keV}$ (a) and $E_{n} \approx 980 \mathrm{keV}$ (b) compared to the data of Smith (blue line). The discrepancies between the different detectors, $\mathrm{LaBr}_{3}$ and HPGe, are caused by the different time-of-flight resolutions and therefore different neutron energy resolutions enabling a finer energy binning for $\mathrm{LaBr}_{3}$.

Eq. (6) the following expression for $W_{\mathrm{n}}$ is derived:

$$
W_{\mathrm{n}}(\theta)=\frac{1+a_{2} P_{2}(\cos \theta)+a_{4} P_{4}(\cos \theta)}{1+a_{2} \underbrace{P_{2}\left(\cos 90^{\circ}\right)}_{=-0.5}+a_{4} \underbrace{P_{4}\left(\cos 90^{\circ}\right)}_{=0.375}}
$$

This function can be used to fit the experimental results to determine the parameters $a_{i}$.

Figure 7 shows examples for the measured normalized $\gamma$-ray angular distribution of the $E_{\gamma}=847 \mathrm{keV}$ transition of ${ }^{56} \mathrm{Fe}$ at an incoming neutron energies of about $930 \mathrm{keV}$ and $980 \mathrm{keV}$. The data are determined within neutron energy bins with respect to the detector's ToF resolution, e.g. in the case of Fig. 7 it is $3 \mathrm{keV}$ for the $\mathrm{LaBr}_{3}$ and $15 \mathrm{keV}$ for the HPGe detector. The solid lines are $W_{n}(\theta)$ functions according to Eq. (7) fitted to the measured data. For comparison the $W_{n}(\theta)$ curve calculated using the Legendre parameters of Smith [26] is plotted. These parameters were determined using quasi-monoenergetic neutrons, which caused a neutron energy resolution corresponding to the width of the neutron distribution of $25 \mathrm{keV}$. The HPGe data nicely re-sample the result of Smith while the $\mathrm{LaBr}_{3}$ data reveal much stronger fluctuations. This becomes even better visible in Fig. 8 where the Legendre parameters $a_{2}$ and $a_{4}$ are plotted over the energy range from the threshold up to $7 \mathrm{MeV}$.

\section{Angular corrections}

Using the results for the angular distribution the cross section data determined in the $\gamma$-ray production and doubleToF measurement can be corrected for angular effects. Instead of assuming $W(\theta)=1$ as was done in Sect. 4 the expression of Eq. (6) with the parameters $a_{2}$ and $a_{4}$ determined in the previous section can be inserted into Eq. (2). In Fig. 9 one can see that the $n$ ELBE data now

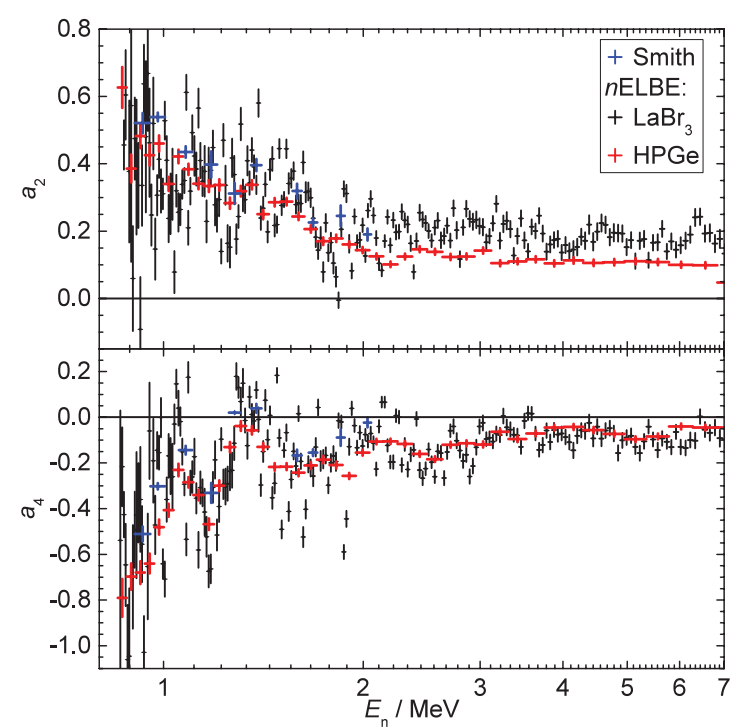

Figure 8. Legendre parameters of the $E_{\gamma}=847 \mathrm{keV}$ transition of ${ }^{56} \mathrm{Fe}$.

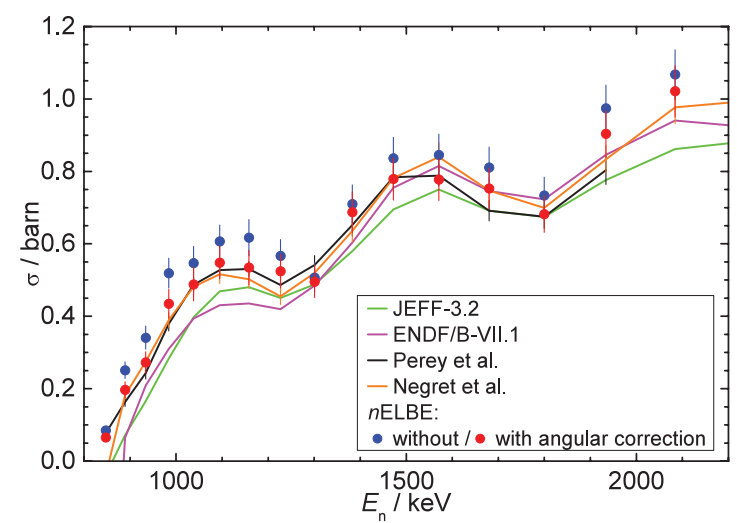

Figure 9. Inelastic neutron scattering cross section under excitation of the first excited state of ${ }^{56} \mathrm{Fe}$ determined in the $\gamma$-ray production measurement before and after correction for the $\gamma$-ray angular distribution. (Note: The reference data are averaged to the binsize of the $n$ ELBE measurement.)

agree very well with the data of Perey et al. [9] and Negret et al. [11]

For the correction of the double-ToF results the much larger angular coverage of the $\mathrm{BaF}_{2}$ scintillators has to be taken into account:

$$
W_{i j}=W_{j}=\frac{\int_{\theta_{\min , j}}^{\theta_{\max , j}} W(\theta) \mathrm{d} \cos \theta}{\int_{\theta_{\min , j}}^{\theta_{\max , j}} \mathrm{~d} \cos \theta}
$$

The range $\left[\theta_{\min , j}, \theta_{\max , j}\right]$ denotes the angular extent of each single $\mathrm{BaF}_{2}$ scintillator, which is in the order of $\left[48-63^{\circ}, 131-117^{\circ}\right]$. In Eq. (8) the assumption is made, that the neutron emission is isotropically and uncorrelated to the $\gamma$-ray. As can be seen in Fig. 10 after application of the $\gamma$-ray angular correction the $n$ ELBE results get closer to the reference data and above $1.6 \mathrm{Mev}$ even agree to some extent. But especially in the range below $1.2 \mathrm{MeV}$ the discrepancy is still more than $10 \%$. This could be due to non-isotropic emission of the neutron or even to $\gamma$-ray-neutron angular correlations, which could be theoretically calculated $[27,28]$ and should be included in a future work. 


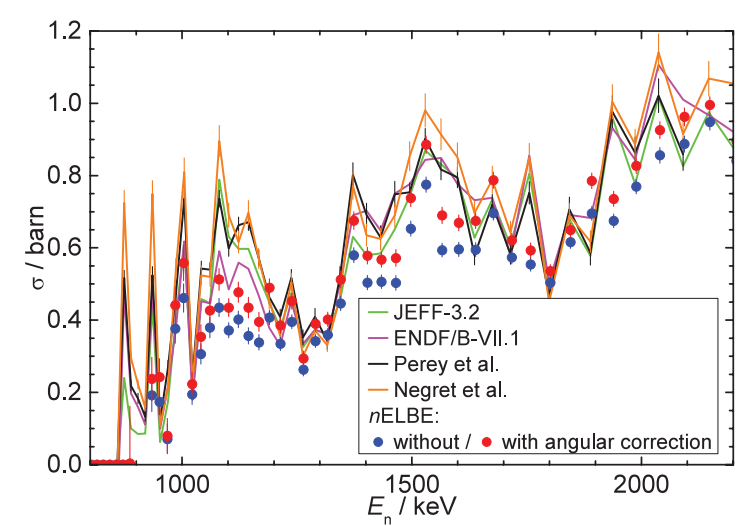

Figure 10. Same as Fig. 9 but the $n$ ELBE data were determined in the double-ToF experiment. In contrast to the photon production data in Fig. 9 the double-ToF data becomes larger after correction, because they were acquired around $90^{\circ}$ relative to the beam and hence are strongly affected by the minimum of the angular distribution.

\section{Summary}

At the neutron ToF facility $n$ ELBE the inelastic scattering on ${ }^{56} \mathrm{Fe}$ was investigated using different experimental methods. The $\gamma$-ray angular distribution was measured with a high neutron energy resolution of about $3 \mathrm{keV}$ using $\mathrm{LaBr}_{3}$ scintillation detectors. The inelastic scattering cross section for the excitation of the $E_{\mathrm{x}}=847 \mathrm{keV}$ state was determined via a $\gamma$-ray production measurement and by means of a double-ToF setup. The results of the $\gamma$-ray production measurement agree very well to data from previous experiments and evaluations. The total uncertainty is in the order of $7 \%$. The results of the double-ToF experiment are on average $10 \%$ below these data, which might be due to non-isotropic neutron angular distribution or even neutron- $\gamma$-ray angular correlations.

We thank the staff of the ELBE accelerator for their cooperation during the experiments and A. Hartmann, K. Heidel and M. Görler for technical assistance. This work was supported by the German Federal Ministry for Education and Science within the TRAKULA project (02NUK13A) and by the European Commission within the projects EFNUDAT (FP6-036434), ERINDA (FP7-269499) and CHANDA (FP7-605203).

\section{References}

[1] M.B. Chadwick et al., Nucl. Data Sheets 118, 1 (2014)

[2] M. Salvatores et al., NEA/WPEC-26 Report No. 6410, ISBN: 92-64-01082-3 (2008)

[3] M. Salvatores et al., Prog. Part. Nucl. Phys. 66, 144 (2011)

[4] OECD NEA Nuclear Data High Priority Request List, http: //www . oecd-nea . org/dbdata/hprl/

[5] J.C. Hopkins et al., Nucl. Sci. Eng. 19, 431 (1964)

[6] W.B. Gilboy et al., Nucl. Phys. 64, 130 (1965)

[7] E. Almén-Ramström, Report AE-503, Aktiebolaget Atomenergi, Studsvik, Sweden (1975)

[8] S.F. Hicks et al., EPJ Web Conf. 93, 02002 (2015)

[9] F.G. Perey et al., Proc. 3rd Int. Conf. Neutron Cross Sections Tech. Knoxville, TN, USA, C, 71 KNOX, 1, 197103, p. 191 (1971)

[10] W.E. Kinney et al., Nucl. Sci. Eng. 63, 418 (1977)

[11] A. Negret et al., Phys. Rev. C 90, 034602 (2014)

[12] F. Gabriel et al., Nucl. Instr. Meth. B 161, 1143 (2000)

[13] J. Teichert et al., Nucl. Instr. Meth. A 507, 354 (2003)

[14] E. Altstadt et al., Ann. Nucl. Ene. 34, 36 (2007)

[15] P. Schillebeecks et al., Nucl. Data Sheets 113, 3054 (2012)

[16] R. Beyer et al., Nucl. Instr. Meth. A 723, 151 (2013)

[17] R. Beyer et al., Nucl. Instr. Meth. A 575, 449 (2007)

[18] D.B. Gayther, Metrologia 27, 221 (1990)

[19] R. Nolte et al., Nucl. Sci. Eng. 156, 197 (2007)

[20] J.F. Briesmeister, Los Alamos National Laboratory Report LA13709 (2000)

[21] S. Agostinelli et al., Nucl. Instr. Meth. A 506, 250 (2003)

[22] JEFF-3.2 evaluated data library, http://www. oecd-nea.org/dbforms/data/eva/evatapes/ jeff_32/

[23] M.B. Chadwick et al., Nucl. Data Sheets 112, 2887 (2011)

[24] R. Beyer et al., Nucl. Phys. A 927, 41 (2014)

[25] M. Dietz et al., these proceedings, EPJ Web of Conf. (2016)

[26] D.L. Smith, Argonne National Laboratory Report ANL/NDM-20 (1976)

[27] G.R. Satchler, Phys. Rev. 94, 1304 (1954)

[28] E. Sheldon, Rev. Mod. Phys. 35, 795 (1963) 